\title{
A Corpus-Based and Complex Computing Digital Media System for 3D Animation
}

\author{
Weihua Zhang $\mathbb{D}^{1}$ and Sang-Bing Tsai $\mathbb{D}^{2}$ \\ ${ }^{1}$ School of Design, Dalian Minzu University, Liaoning 116600, China \\ ${ }^{2}$ Regional Green Economy Development Research Center, School of Business, WUYI University, China \\ Correspondence should be addressed to Weihua Zhang; zhangweihua709@163.com
}

Received 3 November 2021; Revised 23 November 2021; Accepted 1 December 2021; Published 22 December 2021

Academic Editor: Xin Ning

Copyright (c) 2021 Weihua Zhang and Sang-Bing Tsai. This is an open access article distributed under the Creative Commons Attribution License, which permits unrestricted use, distribution, and reproduction in any medium, provided the original work is properly cited.

\begin{abstract}
In this paper, we design a corpus-based 3D animation digital media system to improve the accuracy of 3D animation generation and realize crossplatform animation display. The corpus module extracts high-precision data through web crawling, web cleaning, Chinese word separation, and text classification steps; the character animation generation module uses the semantic description method to expand the frame information description of the extracted data, calculates the object spatial 3D coordinates, and uses the built-in animation execution script to generate 3D character animation; the improved digital media player module uses the improved digital media player to realize crossplatform display of 3D character animations using the improved digital media player. By constructing multidimensional character relationships and combining multiple visualization methods, the complex and multifaceted social relationship network is made available to users in an intuitive and more acceptable and understandable mode. Through a large number of user surveys, it is proved that the visual analysis method combining real social and virtual social proposed in this paper provides a more adequate and reliable basis for friend recommendation and social network analysis; the combination of multiple character relationships with geographical information and the use of visualization to describe multidimensional historical character relationships provides a new research perspective for the research and exploration of humanistic neighborhoods. The experimental results prove that the designed system can effectively read known contents and extract keywords and generate 3D animation based on keyword features, with a high accuracy rate, fast response time, small frame loss rate, and crossplatform display animation advantages.
\end{abstract}

\section{Introduction}

Digital media art is a multidisciplinary and crossdisciplinary art form, a connection between art creation and science and technology. Advances in technology have given birth to new art forms, from film, television, and video to video art, multimedia art, and digital art, all of which have been inspired by advances in science and technology to give birth to new art. The advancement of technology has given birth to new art forms, from film, television, video to video art, multimedia art, and digital art, all of which are the advancements of science and technology that have inspired the birth of new art. Advances in science and technology have created new platforms and spaces for artists, greatly enriching the forms of artistic creation and promoting the development of art.
Digital media art is the establishment and expansion of new fields of art supported by new science and technology, such as digital newspapers, digital film, three-dimensional virtual space, and mobile digital media. It has become an excellent way to develop new fields of art. It is digital media art that disseminated across media and flush with special art forms and language patterns and is gradually being understood and used by increased people. As time goes by, digital media art gradually penetrates our daily life, and it is not only new technology but also a new way of life and aesthetic form [1]. Along with the rapid development of science and technology, the application of digital media art is becoming increasingly widespread. The application of digital media, a new form and novel technology in art, has a broad development prospect and market appeal and is flourishing, but 
because the country is in its infancy, the related theory and technology are not perfect and mature [2]. In this context, this thesis takes digital media art as the research object, deeply studies and explores the application cases of digital media art in display design, and summarizes the relevant theoretical exploration and forward-looking prediction for the future.

Technology has led to the constant updating and development of digital media, and the traditional static sculpture is no longer enough to satisfy the artist's expression of sculptural language. Sculptures do not only stay in their practicality and decoration, but nowadays, they tend to be more interactive, and sculptures show their way of thinking in a new way without missing the real connotation of sculpture [3]. Digital developments have allowed sculptures to take on more forms than just the traditional practical sense. Traditional art and new technology are gradually coming together, as exemplified by the birth of CG illustration, which has infiltrated animation, games, film, and television. Digital sculpture and 3D character animation are two disciplines in which 3D technology is applied, and they are both different and related [4]. It is a digital media art that spreads across media and is equipped with special art forms and language models. It is gradually being understood and used by increased people. During the dissertation research, the following sections are covered: the comparison between sculptural modeling and 3D character animation; the connection between digital sculpture and 3D character animation; and the exploration of mutual learning and enhancement among $3 \mathrm{D}$ character animation and digital sculpture. The difference between the two art forms in terms of practice makes the research on the correlation between them relatively scarce, the relevant researchers at home and abroad are relatively scarce, and the relevant literature is also relatively scarce. Only a few people have explored the integration between the two disciplines of digital sculpture and 3D character animation. The study of this paper requires strong professional skills and comprehensive professional ability [5]. Firstly, to explore the relationship between the two, as a basic research, one must understand the background, purpose, significance, relevant software and theoretical basis of the topic, and other basic theories. Secondly, find the similarities and differences between digital sculpture and 3D character modeling and find the intrinsic connection between the two based on the similarities. Analyze how the two can learn from and improve each other based on their correlation.

Corpus is a large-capacity electronic text database containing language fragments or texts constructed by following the laws of fixed linguistics. It can analyze a large amount of linguistic data by computer and elaborate the real situation of data usage from a macroperspective, with high accuracy of data analysis. To improve the accuracy of 3D animation and realize the crossplatform display of animation, this paper designs a digital media system of 3D animation based on the corpus. The experimental results prove that the system in this paper can effectively improve the accuracy of data extraction, successfully build 3D animations, and can displayed on different platforms. Cartoons are a very popular form of entertainment for children, and cartoons are even more closely related to the growth and development of young children. By focusing on the composition of the language of children's cartoons, this paper hopes to draw the attention of more children's animation script creators, who can combine the language development characteristics of children while creating cartoons, so that children's cartoons can be suitable for children's growth and development.

\section{Current Status of Research}

In recent years, animation design has been developed rapidly, and at the same time, the quality of $3 \mathrm{D}$ animation material design has also put forward higher challenges [6]. This has put forward new requirements for the work of the 3D animation material management system platform. At the end of the last century, with the development of China's Internet business, the issue of China's domestic design informatization was widely mentioned, as a kind of information system for the design process resource optimization allocation as the main development goal is the process of acquiring, processing, and regenerating the information formed in the design process [7]. The Informa ionization of design resource base management is also one of the key elements that come out in this process [8]. Design resource library management informatization is this in the design process, the existing design information, and design resources through the application of many Internet technology and multimedia technology to achieve its overall planning [9]. It can analyze a large amount of language data through a computer and explain the real situation of data use from a macro perspective, with high data analysis accuracy. For the development of this system, many companies engaged in the design of three-dimensional animation materials have formed a more complete resource management system; at the same time, most design companies through self-development or outsourcing on line based on WEB technology threedimensional animation material management information system basically have realized the demand for network resources for the management of the design system [10]. In the process of promoting the information construction of animation material management, to break through the barriers of core technology, it must achieve the normality of information in the process of use, the flexibility, and development of the system, etc., which will directly affect the steady progress and rapid development of the information construction work of the three-dimensional animation material library resource management.

The effect of the application of $3 \mathrm{D}$ animation material management system is more obvious, among which the more prominent is the design process drawing management system represented by PTC, which can easily view the design drawings in the library and can realize $3 \mathrm{D}$ preview and $3 \mathrm{D}$ to $2 \mathrm{D}$ conversion, which greatly facilitates the design department to carry out the design management of drawings [11]. Aydinl et al. designed an animation interactive platform system based on microcontroller and Scratch, which connects the real world with the animation world by combining pure computer-side animation programming and lower computer programming, with the advantages of development and sharing [12]. Chollet et al. designed a 3D animation production assistance system 
based on visual recognition, which uses the motion and expression capture technology in visual recognition to render the animation task action expressions, which improves the efficiency and quality of 3D animation production [13]. Most design companies currently use three main types of 3D animation material management platforms, stand-alone management mode, departmental LAN management mode, and company LAN management mode. Among them, the standalone management mode and the departmental LAN management model require a high degree of data processing, due to the lack of effective sharing between the drawing resources of each designer; so, the designers often have the problem of repeated design in the process of material design [14]. It seriously restricts the overall material drawing design efficiency of the enterprise. Therefore, in this paper, we concentrate on a management information system based on the company's local area network. In this system, the main purpose is to provide the necessary information services for the whole company by fully utilizing the data of each department through the integration of multiple departmental management systems [15].

$3 \mathrm{D}$ animation material library platform is a database management software developed for better management of $3 \mathrm{D}$ animation material resources. According to the actual requirements of the design company, that is, to achieve the basic information of designers, 3D animation material resource information, material map review, and sign and material map version management as one of the centralized managements of $3 \mathrm{D}$ animation material, the $3 \mathrm{D}$ animation material database platform can help the designers to manage these tedious data easily and quickly. It can record in detail the records of the designers every time they log in to modify and can identify different versions of the material drawings, which effectively improves the problems of information loss and version confusion in the design process. The 3D animation material library platform has advantages that cannot be compared with manual management: easy to find, high reliability, good confidentiality, and low cost.

\section{3D Animation Corpus Digital Media System Design Analysis}

3.1. 3D Animation Corpus Rationalization. With the development of the times and the progress of science and technology, information technology has completely changed people's lives, and concepts such as information systems, which are closely related to it, are rapidly attracting people's attention. As people know increasingly about information systems, they realize that information system is the combination of processes such as collecting, transforming, and saving data, and its main role is to help people do their work more easily and quickly and solve practical problems through the operation. By paying attention to the language composition of children's cartoons, we hope to attract more children's animation script creators' attention. While creating cartoons, we can combine the characteristics of children's language development to make children's cartoons truly suitable for children's growth and development. By studying the definition of information, people realize that only under certain conditions can data be transformed into information, and only after corresponding processing and transformation can data be adopted as information and can become effective information and help people to solve practical problems [16]. It is also important to note that processing and transforming information resources are an essential feature of information systems, and only after corresponding processing can data fully meet the requirements of existing procedures and thus become truly qualified information that can have a significant impact on decisionmaking. It is on this basis that it is shown that data is the factor that plays a fundamental role in the process of decision management. At the same time, in the process of working with information systems, the talents of the designers are fully demonstrated, and the participation of each staff member realizes his or her technical skills. It is in this way that the information system can make the best data integration and can help in the final decision making.

The corpus-based 3D animation digital media system is divided into 3 modules, which are corpus module, character animation generation module, and improved digital media player module, and the system block diagram is shown in Figure 1.

A corpus is a collection of linguistic materials collected and sampled using certain principles and classified and assembled by certain methods for a certain purpose, which has the advantages of a large amount of information, a strong representation of linguistic materials, and a wide range of applications. The data collection phase is to find the hypertext markup language (HTML) pages related to the topic on the Internet and store them in the initial web page library [17]. The main function is to help people complete their work more conveniently and quickly and solve practical problems through operations. Through studying the definition of information, people realize that only under certain conditions can data be transformed into information and only after corresponding processing and transformation. The page cleaning stage is to clean and optimize these HTML pages, which must be changed from different page encoding to the same encoding, because there are many pages with different encoding on the Internet, and finally, these HTML pages with the nonstandard structure are changed to standard Extensible Hypertext Markup Language (XHTML) pages, which are stored in the clear page library. The page parsing process is to use Extensible Markup Language (XML) parser to analyze these standardized pages, remove the information in the pages that are not related to the topic, obtain the data related to the topic, and store these data in the corpus. The word separation phase is to implement Chinese word separation on the acquired data to get the word frequency and document frequency. The category core word acquisition phase is responsible for calculating the core words of each category and ranking them according to their importance. The scale control phase is to repeat the above process through the acquired category core words until the scale meets the requirements.

$$
\frac{Q\left(Y / V^{*}\right)}{Q\left(Y^{*} / V^{*}\right)} \geq Z
$$




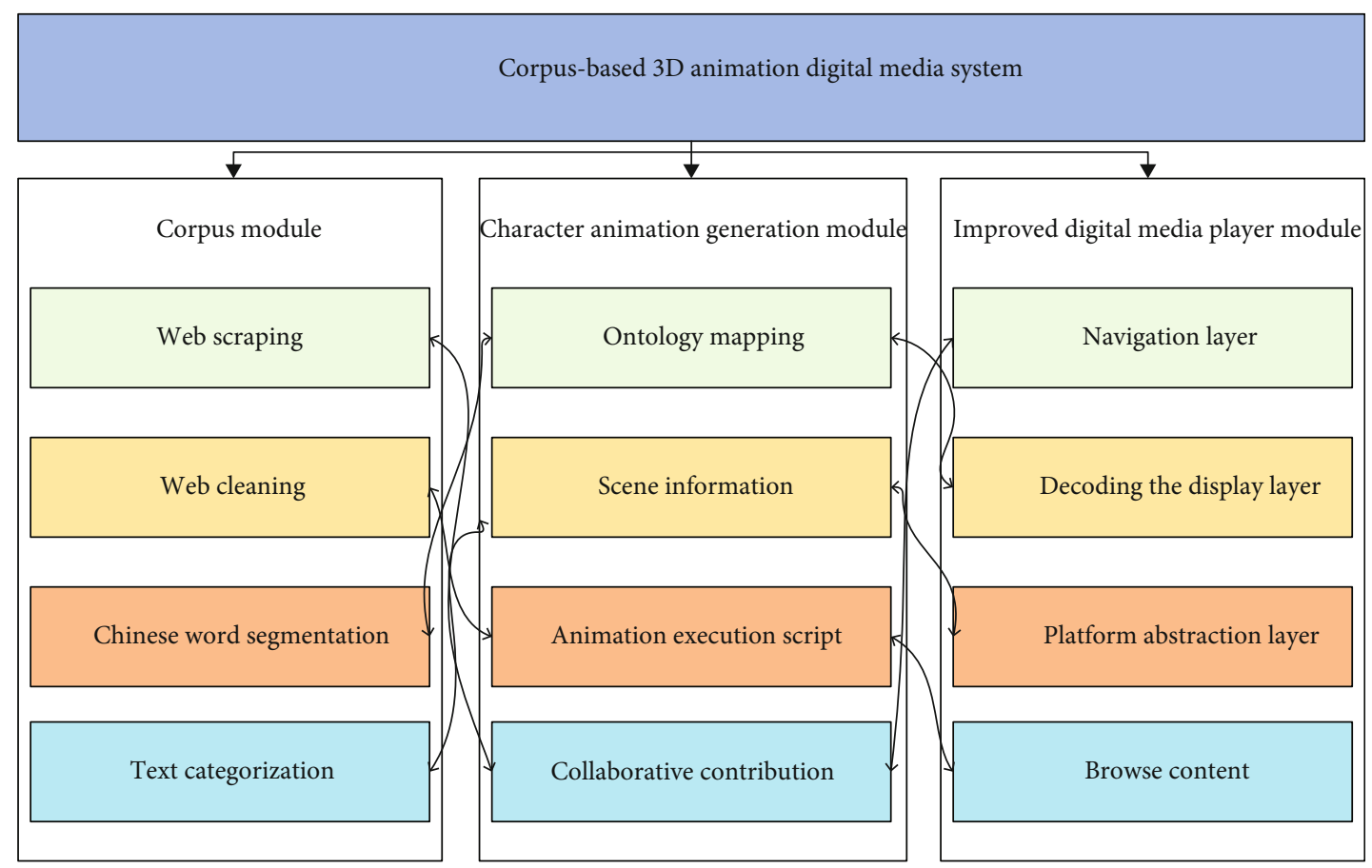

Figure 1: Corpus-based digital media system for 3D animation.

Digital sculpture and three-dimensional character animation belong to the same category of three-dimensional art and also follow the principle of the whole and the local. Different creators have different degrees of understanding of the whole, whether it is painting or 3D art, and they all follow a process of first the whole, then the local, and then from the local to the whole. In the early stage of modeling, we should learn to "give up" the details, so that we can "get" the accurate shape, according to the accurate shape, and then carry out the in-depth portrayal of the details. When dealing with details, you still must consider the whole, to avoid the situation of overwhelming the main. When the details are deeper, we generally must adjust the whole, rather than continue with the details. In this way, the character models and digital sculptures of $3 \mathrm{D}$ character animation are stylistically rigorous without losing the exciting details. The idea of the whole permeates all art classes, and for works of realistic character, all visual images created are portrayed with nature as the original form.

$$
\begin{array}{r}
\frac{s g\left(Y, V^{*}\right)}{S N} \leq Z, \\
\ln \frac{Q\left(Y / V^{*}\right)}{Q(Y) Q\left(V^{*}\right)} \leq Z .
\end{array}
$$

In the digital age, digital technology has brought about tremendous changes and impacts on social life, spirituality, and economic life. At the same time, the development of digital art has also led to the development of digital sculpture and $3 \mathrm{D}$ character animation, neither of which is a completely independent entity between the industries, yet displaying their own unique and individual characteristics
[18]. Digital sculpture and 3D character animation, as two directions of digital art born by relying on digital technology, have the same era, representativeness, and humanity as the products of digital art, although they are studied in different directions.

$$
\operatorname{sim}\left(v_{i}, v_{j}\right)=\frac{1}{\mathrm{JS}\left(v_{i}^{2}, v_{j}^{2}\right)}
$$

The biggest advantage of using network display is that it can be free from the limitation of time and space, and everyone can be at the home, office, and any other place with the network, if there is a demand for knowledge, just open the page through the computer. Using online display, so that the display becomes ubiquitous, the audience can just click the mouse keyboard in front of the computer, and the network display of this powerful advantage will also be the inevitable trend of future display design. The online display makes full use of the Internet's wide coverage, fast dissemination characteristics, while the network display also not limited by time and space. Online display to convenience for people to contact, more vivid form is gradually adopted by display designers, and at the same time, this can also meet people in the new era of the new requirements of the display.

$$
\mathrm{JS}\left(v_{i}^{2}, v_{j}^{2}\right)=\frac{1}{2}\left(D_{\mathrm{KL}}\left(v_{i}, v_{j}\right)-D_{\mathrm{KL}}\left(v_{j}, v_{k}\right)\right) .
$$

The database of the $3 \mathrm{D}$ animation material management system is using the SQL Server2000 database, the database operation is relatively simple, and the $3 \mathrm{D}$ animation material 
management system such as small and medium scale system can provide faster data access. The program of $3 \mathrm{D}$ animation material management system is written in java language, it uses S2SH framework, separating various business separation techniques within the source system is the feature of S2SH framework, making the system run more stable and easier for the later maintenance of the system, and the front of 3D animation material management system uses CSS + DIV, guaranteeing the dynamicity and stability of the page and finally the $3 \mathrm{D}$ animation material. The debugging operation of the management system is very convenient and stable.

$$
D_{\mathrm{KL}}\left(v_{i}, v_{k}\right)=\sum_{k=1}^{K} p\left(z \mid v_{i}\right) \ln \frac{p\left(z \mid v_{i}\right)}{p\left(z_{k} \mid v_{j}\right)}
$$

The 3D animation material management system is completely developed and designed for enterprises, and none of the content involved in the development process involves contracts, responsibilities, and other aspects that contradict the law. First, in the standard configuration of the desktop computer and ordinary browser can run normally on the three-dimensional animation material management system, the enterprise's desktop computer can run normally. The air mouse is the air mouse. When the user holds the air mouse and swipes it, the sensor inside the air mouse will sense the change of gravity, thus realizing the function of position sensing. There are specially designed air mice, and some are integrated in the remote control. Touchpad control generally involves designing a touchpad on the TV remote control, and some are specially equipped with a touchpad. The principle is to use the sliding operation of the finger to move the input device of the cursor. The operation is like that of a laptop. Gesture somatosensory control currently generally captures the user's gestures and body movements through a camera and realizes control through present action corresponding functions. Users can click anywhere on the screen, which will bring convenience to many operations such as games, web browsing, and video-on-demand. In addition, air mouse can also support many applications and games for motion control, making it more fun for users to use. Changlong's air mouse also has a voice button. If the user does not respond sensitively to the TV when making a far-field voice, he can press the voice button on the air mouse to perform voice operations. The category core word acquisition stage is responsible for calculating the core words of each category and sorting them according to the importance of the core words of these categories. The scale control stage is to repeat the above process by obtaining the core words of the category until the scale meets the requirements. Second, the people who can access the system are the designers and administrators within the enterprise; so, for the utilization of resources and operability, only a small number of tables in the operation database need to be operated, and it can make the three-dimensional animation material management system complete and stable operation, without causing huge pressure to the three-dimensional ani- mation material management system. Third, the operation of the three-dimensional animation material management system is relatively simple, and the UI interface design is more humane, whether it is a manager or designer can be smoothly operated, not only will not affect the internal work effect of the enterprise but also provides some more humane features to help improve efficiency, as shown in Figure 2.

Functional requirements analysis is the basis of $3 \mathrm{D}$ animation library management software. Through functional analysis, we can realize the basic content of 3D animation library management software. Specifically, there are common functions such as managing $3 \mathrm{D}$ animation material, maintaining, and managing system stability functions, managing user functions, and managing retrieval functions and other less common functions. These functions are the focus of the design and development of the $3 \mathrm{D}$ animation material library management software. Resource management targeted at $3 \mathrm{D}$ animation clips to ensure the availability and reliability of these $3 \mathrm{D}$ animation clip resources. In fact, 3D animation material management is mainly aimed at uploading and downloading many existing 3D animation materials, as well as querying and deleting, modifying functions, etc. At the same time, users have different kinds and characteristics. Therefore, in uploading and downloading, just a single resource downloading and uploading is not enough to satisfy users' needs, but more to realize the uploading and downloading function of multiple resources at the same time. In addition to this, there is a need to provide the ability for users to self-describe the resources in the system themselves and to be able to use keywords for searching and accessing them. There is also a need to provide functions such as modifying and deleting keywords.

$$
v_{k}=\frac{1}{2}\left(v_{i}-v_{j}\right) .
$$

The ability to find and use resources must not only have a detailed record of the resource but also the ability to read specific information about that resource promptly $[18,19]$. This condition must be fulfilled to ensure the needs of the users. Because in a system, there are undoubtedly many information resources, and how to find the information that is useful to them and make use of it among the many information resources is the goal of the user. So according to this need of the user, there must be a perfect mechanism for the user to find and browse to provide convenience. In addition, the $3 \mathrm{D}$ animation material library management software must also achieve online interactive functions, not only to enable designers to query the database resources more easily. Communication and review functions should also be designed to allow managers to review the material design drawings in the database and ensure that these reviews are recorded and made visible to other users.

3.2. 3D Animation Digital Media System Design. As a continuously running system, the functions of the $3 \mathrm{D}$ animation material library management system are to enable the 


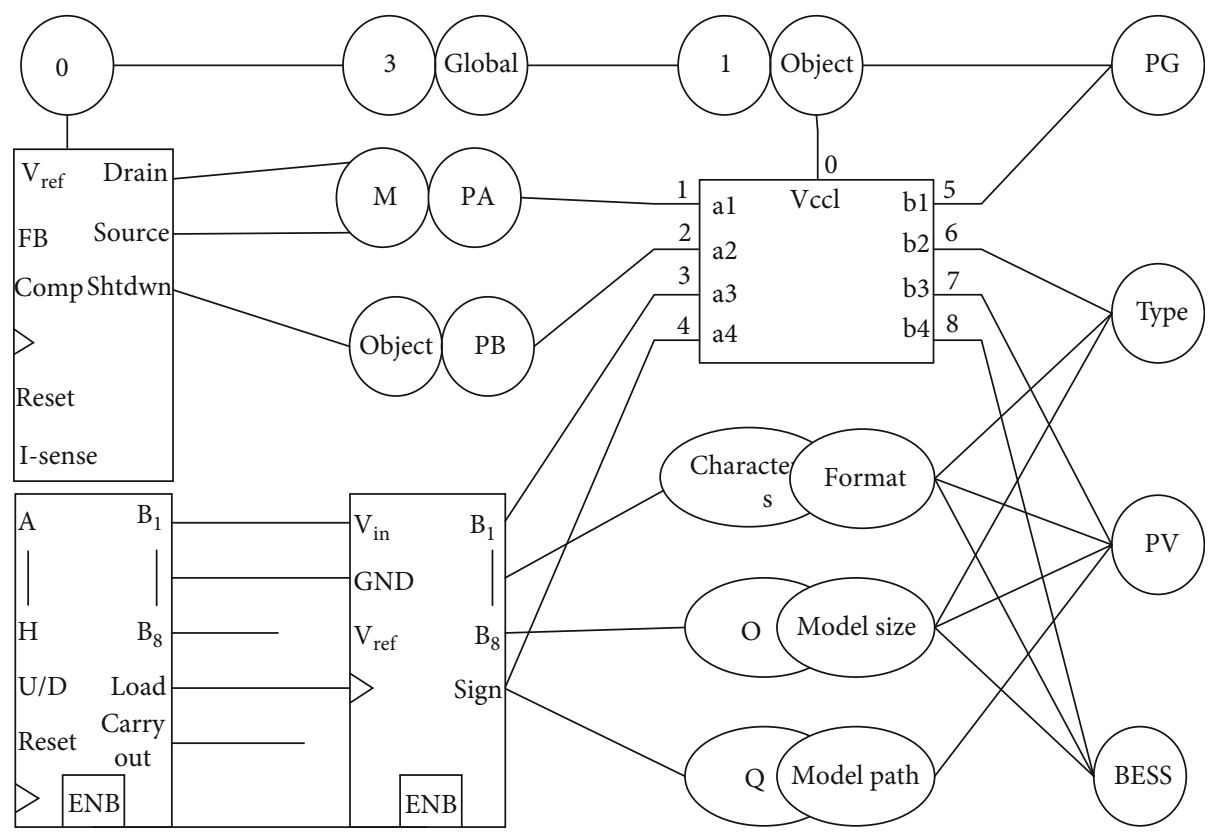

Figure 2: Model ontology library.

management and maintenance of itself in addition to the users and data. The purpose of managing itself is for the maintenance of the system, its stability, and scalability and supports for user access. These maintenance functions should be able to both ensure that the system is backed up in the event of an unstable state and to achieve system emptying and recovery functions to ensure normal operation of the system.

Users of the 3D animation footage library include different designers. Their use of resources is mainly focused on uploading and downloading 3D animation footage resources, as well as querying and deleting, modifying, and other functions $[20,21]$. Whether it is painting or threedimensional art, it follows a process from the whole to the part and then from the part to the whole. In the early stage of modeling, it is necessary to learn to "let out" the details, to "get" the accurate shape, and then carry out the indepth description of the details according to the accurate shape. At the same time, the users have different kinds and characteristics. Therefore, in uploading and downloading, just a single resource downloading and uploading is not enough to satisfy the users' needs, but more to realize the function of uploading and downloading multiple resources at the same time. In addition to this, there is a need to provide the ability for users to self-describe the resources in the system themselves and to be able to use keywords for searching and accessing them [22]. There is also a need to provide functions such as modifying and deleting keywords. The system should also give different permissions to different users, for example, administrators can have the function of uploading resources, while designers have the function of downloading and browsing to ensure that the management and security of the system resources are achieved.
The design focus of the 3D animation material management system is the functional design of the system. The basis of the 3D animation material management system is the functional design, and only if the functional design is complete can the normal operation of the system be realized. Not only should the design be considered from the designer's point of view but more importantly from the user's point of view, because only in this way can the normal operation of the functional board be realized, such as how to manage database resources, how to manage users, and how to manage the system and maintain the system and the retrieval function. The functions designed through different perspectives can only make the platform a perfect platform; that is, there is a system platform for the design manager, and then there is also a $3 \mathrm{D}$ animation material platform for the users. Both as the management platform of the system bear different roles and functional requirements, respectively, and its module framework is shown in Figure 3 below.

The more important aspect of the $3 \mathrm{D}$ animation material management system is the design and management of permissions in access control management. By limiting the scope of the resource management object, restrict all designers to freely access their management functions outside the three-dimensional material library resources; of course, the resources cannot be open to all designers and allow all designers to design planning resources. To achieve this restriction, the general designer is in the access rights that are divided into two access modes of authentication login type and nonauthentication login. For these two access modes, different user identities can implement different access methods. For example, a registered user of the system can achieve authentication type access. And nonregistered users can implement a nonauthenticated access mode. The 


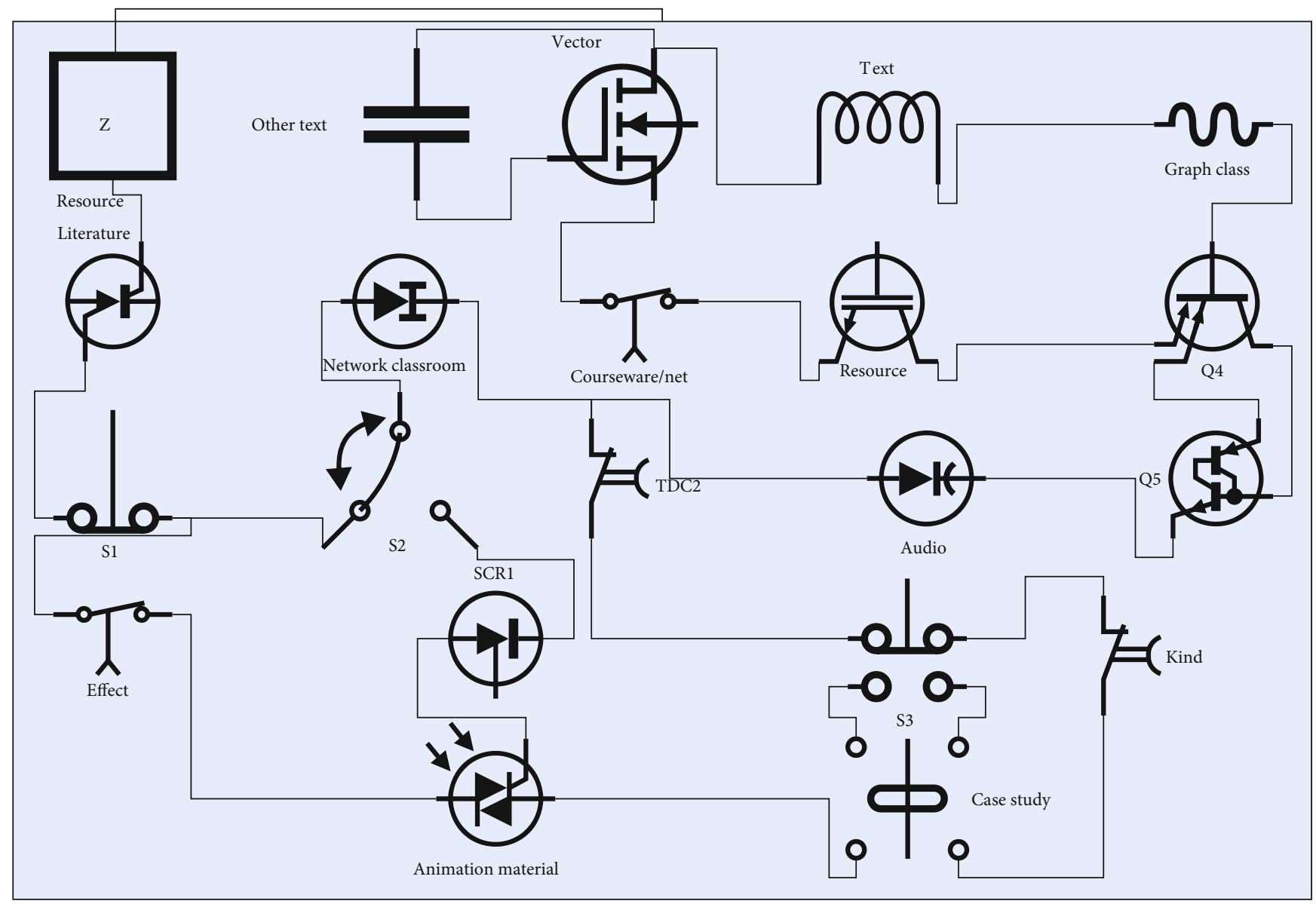

Figure 3: Resource classification map.

development of digital art has also led to the development of digital sculpture and three-dimensional character animation. The industries are not completely independent individuals, but they show their own unique and individual characteristics, as shown in Figure 3.

Take managers as an example, user logins for general managers are divided in two ways: full selection and unit selection. Comprehensive selection is to grant certain permission all to a team of managers, while unit selection is to subdivide the permission, and on this basis, according to the different characteristics of different managers, respectively, authorized. Statistical analysis and management can effectively play a role in enhancing the utilization rate, as a platform for the $3 \mathrm{D}$ animation material management system to achieve the coordinated development of various parts of the function. The design function of the statistical analysis module cannot only be used effectively for resource management of 3D animation material. And as an important function of platform resources, it can also effectively carry out statistical analysis of resources and detailed statistical resource storage management. 3D animation material management system with the help of statistical analysis not only can the system of resource type, resource quantity, and effectively improve the statistical analysis of storage uti- lization. Moreover, the most important thing is to track the access records of designers, as well as to realize the hot access tracking of system resources, and finally, the results of monitoring are gathered into statistical information icons.

The ultimate purpose of classification is to perform storage, which is the core work of database resource management. This is the goal of managing textual and documentary information. Therefore, the way of classifying these different categories of information becomes the key factor. For this, a data structure approach is used. This is shown in Figure 4. The ultimate purpose of classifying the database resources is for storage, and this is the core technique of resource management database. With the help of this technique, effective management and querying of textual information, as well as various management documents by selecting some way, should be the goal of every database design. See Figure 4.

The main function of this section is the functional definition and input of information. It is about the design of what kind of resources and how they are to be entered into the system. The issue of data quality in input design is critical and needs to be given full attention. Based on this, the search terms must be set correctly. If the input search terms 


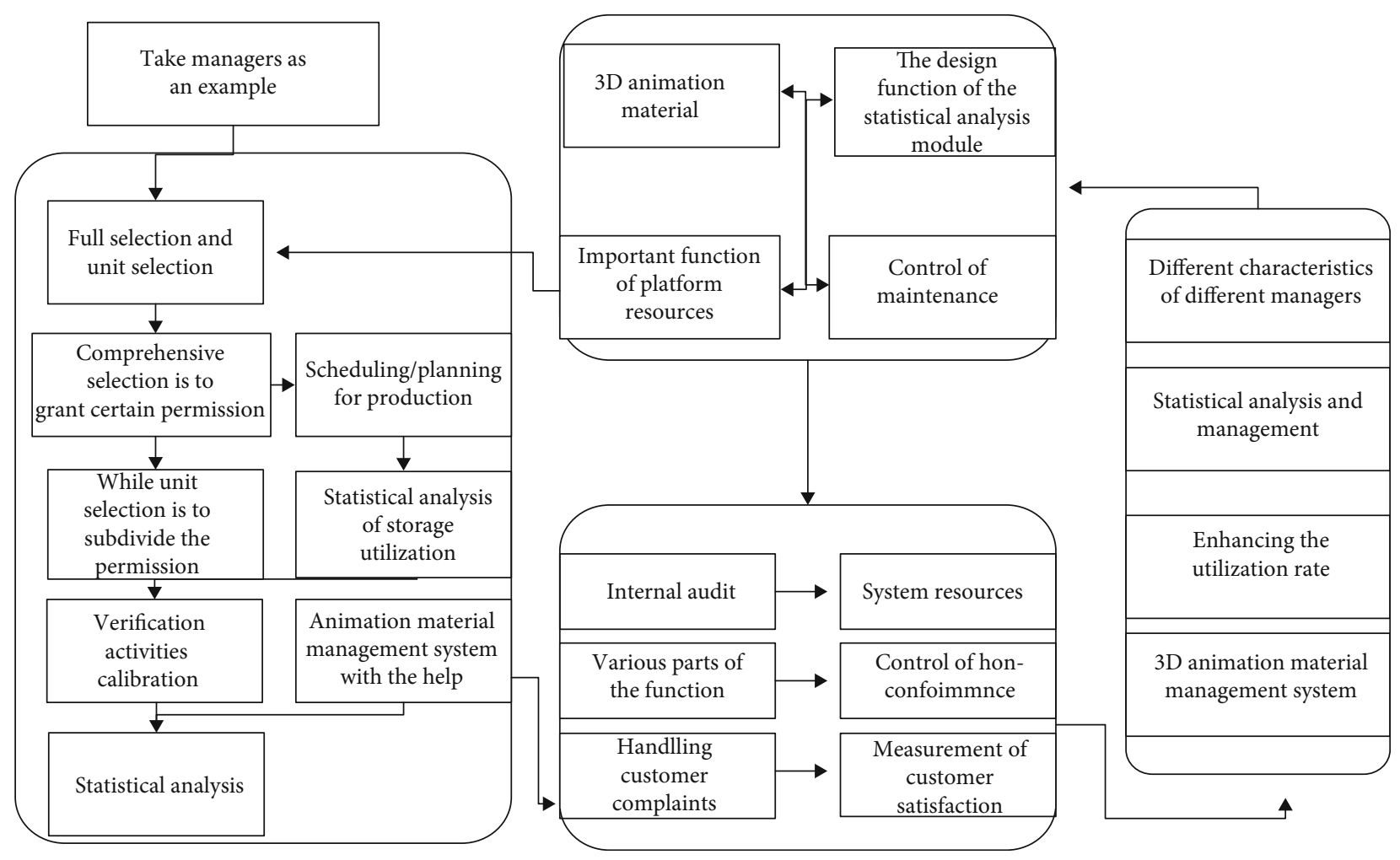

FIGURE 4: Diagram of the relationships between the entities in the system.

are not highly relevant to the original data information, even if the system technology is advanced, the search tools matched, the system performs well, and the final search results will be very different from the user's needs, which will directly affect the evaluation of the system effectiveness. This powerful advantage of network display will also be the inevitable trend of future display design. Online display makes full use of the wide coverage and fast spread of the Internet. At the same time, online display is not limited by time and space. In addition, as an interactive state, poor input design will directly affect efficiency. The design specifications used for the two phases of the data input and data preparation process refer first to the criteria for data input, while the data preparation process, in which a series of surveys and statistics must be conducted, is equally important. Because the statistics usually must contain information from multiple designers, this information directly reduces the number of data revisions required to reduce the data, circumventing the workload of tedious repetition, or direct changes to the original data. If allowed, it can also be opened to managers, allowing the data to be at their level is to effect change.

\section{Analysis of Results}

4.1. Experimental Analysis. Taking a part of a selection from a children's storybook as an example, the system in this paper is used to generate a $3 \mathrm{D}$ animation of this content. The system in this paper can effectively read the known con- tent and extract the keywords, generate the 3D animation based on the features of the keywords, and display it through a digital media player. Recall refers to the ratio of the number of relevant documents searched by the system to the number of all relevant documents in the document library and measures the search completion rate of the system search. The accuracy rate is used to measure the accuracy of the system in generating 3D animated characters. For this purpose, the system in this paper is used to extract data with microcontroller and Scratch-based animation interactive platform system and visual recognition-based $3 \mathrm{D}$ animation production assistance system and compare the accuracy and recall rate of data extraction of the three systems, and the test results are shown in Figure 5.

According to Figure 6, it is found that the average accuracy of data extraction of this paper system is $96.7 \%$, the average recall rate is $92.7 \%$, while the average accuracy of the remaining two systems is $84 \%, 84.9 \%$, and the average recall rate is $82.2 \%, 82.6 \%$, respectively. The experimental results prove that the system in this paper has high accuracy in data extraction and can accurately provide data support for the subsequent operation.

The third is that the operation of the $3 \mathrm{D}$ animation material management system is relatively simple, the UI interface design is more humane, and both managers and designers can operate smoothly. The performance of the player of this system is tested, and the performance of the player is mainly divided into three aspects: start-up time, frame loss rate, and closing time. The start-up time 

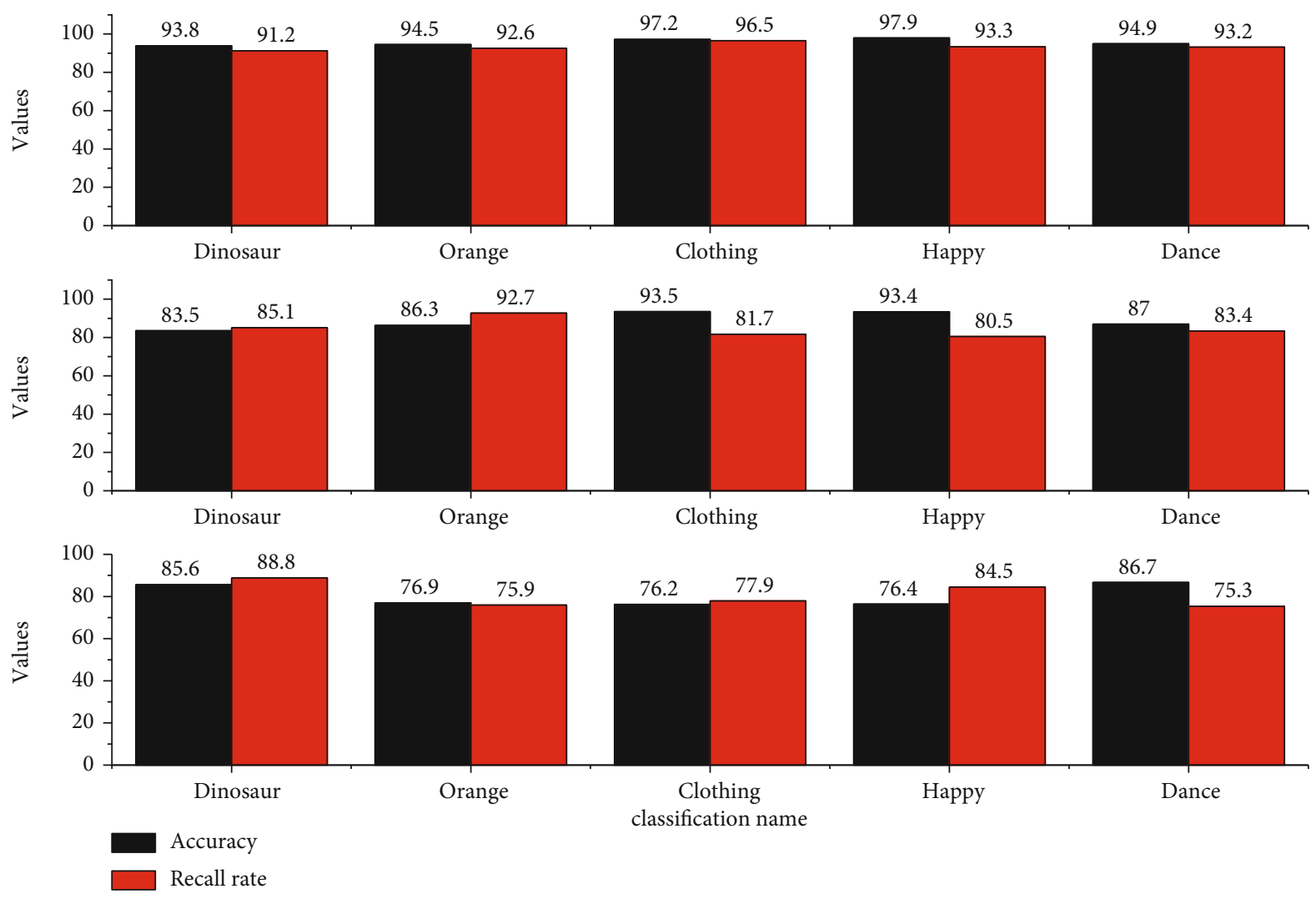

Figure 5: Accuracy and recall of data extraction.

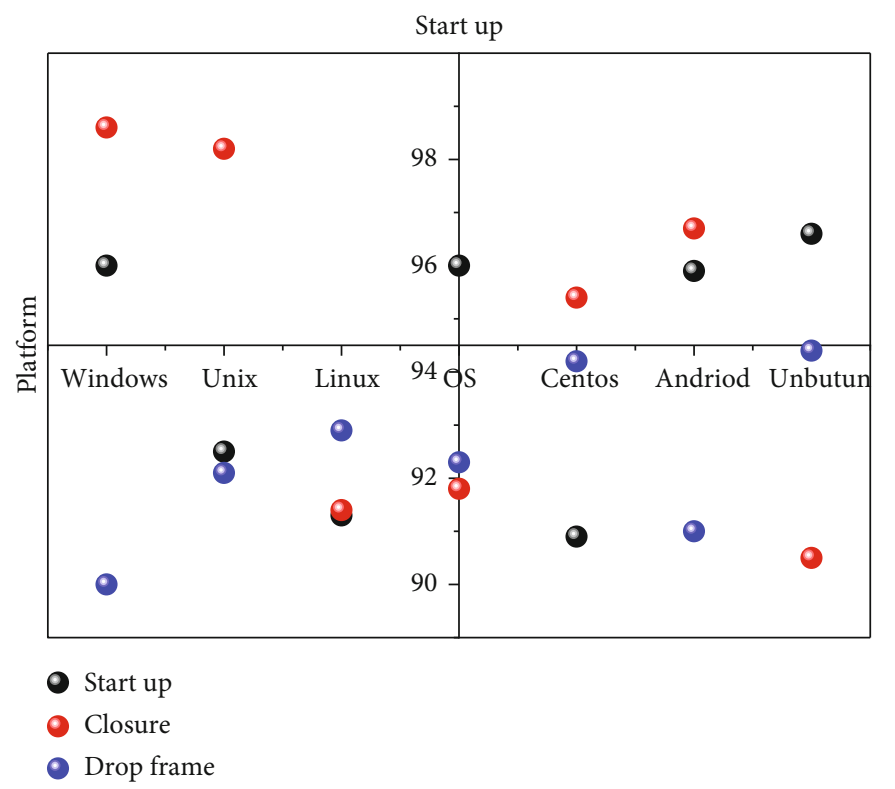

FIGURE 6: Playback performance of the system on different platforms.

represents the time from opening the $3 \mathrm{D}$ animation to the successful display of the animation on the device, the size, format, and resolution of the $3 \mathrm{D}$ animation file, and the hardware performance of the platform will affect the startup time; the frame loss rate represents the percentage of the dropped frames to all the frames, and the decoder is overloaded so that the $3 \mathrm{D}$ animation image cannot be received outside the renderer soon enough to cause the frame loss rate; the shutdown time represents the time from the player receiving the shutdown command to the 


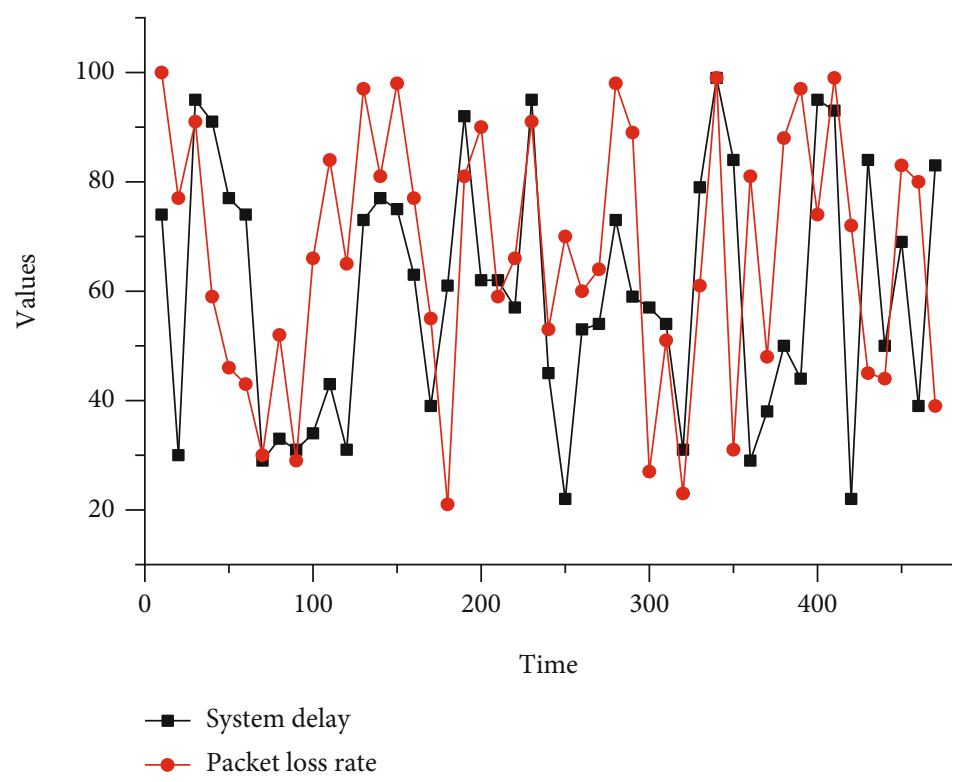

FIgURE 7: System performance test results.

complete. The shutdown time represents the time from the player receiving the shutdown command to the complete shutdown. The $3 \mathrm{D}$ animation designed in this paper is represented by wmv1, and the $3 \mathrm{D}$ animation obtained from the network is represented by wmv2. Figure 6 shows the performance test results of this system on different platforms. According to Figure 6, the start-up time, frame loss rate, and closing time of the two test files played by the player of this system on different platforms are similar, which are within the acceptable range. In the case of increasing test files and resolution, the performance of the player of this system does not degrade significantly and is still within the acceptable range. The experiments prove that the system can display $3 \mathrm{D}$ animation on different platforms with a fast response time and small frame loss rate. See Figure 6.

The four work areas of system planning, system analysis, system design, and system implementation are included in the complete $3 \mathrm{D}$ animation material management system and are part of the management information. The logical and technical design aspects of the functional issues are mainly solved by the system analysis and system design work sessions, while the technical transformation of the design into a realistic $3 \mathrm{D}$ animation material management system is achieved physically by the system implementation of the past work results. In uploading and downloading, only the downloading and uploading of a single resource cannot meet the needs of users, and more is to realize the uploading and downloading function of multiple resources at the same time. The system implementation helps to maintain the reliability of the system, and on the other hand, to achieve the maintainability of the system.

The test process then proceeds, the input modules are tested, and then the resulting passes are scrutinized. Since the modules meet the requirements specified in the above procedure, see if they also meet the intended purpose. If so, then the system design is correct. If not, then the system design is incorrect. At this point, the input modules need to be checked step by step, considering the special data and the exact time of calculation storage, to lock in the error as much as possible and find out where the error lies. The joint adjustment stage is a kind of stage to test the organic union of each module, and the test subject is still the overall system design while including some of the modules, the interface of each module, and the function interface, where the test angle of the function interface is its data transferability function, and finally get the error of each module in data transfer. The joint adjustment phase and the system debugging phase have different debugging degrees, and the former debugging perspective is the system, while the latter debugging perspective is the parts of the system.

4.2. System Performance Results. In the 3D animation material management system, one of the key indicators of how fast or slow the mass sending is the access process, and the process of software testing is the most important. To see the performance load of the system, information processing capacity, and the integrity of sending, we conducted a perfect stress test; that is, we used concurrent login operation to send many SMS contents in a short period, and at the same time, we judged the fast and slow mass sending by recording the contents and number of SMS messages sent out and checking the omission of the receiving end. Observing Figure 7, we can find that when the number of mass SMS is greater than four hundred, the packet loss rate starts to appear and rises slowly; when the number of mass SMS is greater than five hundred, the packet loss rate rises rapidly, and the data also starts to be delayed. Thus, it can be concluded that to maintain the sending performance of the system and avoid system delay, the mass sending volume of the data of the 3D animation material management system developed in this paper should not exceed 400 as much as 
possible, and if a similar situation occurs, a buffer zone can be set aside at the SMS server side, and a queue of user accesses can be constructed in the buffer zone so that the mass sending volume of the data is always kept below the peak. Of course, if the system is improved on its original basis, it can further meet the needs of efficient various departments for system performance. See Figure 7.

In the face of different display content, touch screens can be added to the display design to make the information more flexible and relevant. Touch screen technology has been quite mature at present, in the display design will also replace the traditional display board, and the touch panel area will also become larger. In the display design, the touch screen is mostly used to embed into the wall, and this practice can improve the space utilization rate and can strengthen the connection between virtual space and real space, which is conducive to the dissemination of information. In the display design to join the voice assistant, by transmitting the visitor's voice to the computer, the computer will voice information processing, make the corresponding response, and enhance the fun and interactivity of the display equipment. The purpose of self-management is for system maintenance, system stability, and scalability and to support user access. These maintenance functions must not only ensure that the system is backed up when the system is in an unstable state but also to realize the system's emptying and recovery functions to ensure the normal operation of the system. With future technological advances, the application of various sensors will enable people to operate and interact with more than just the use of voice, touch, eyeballs, etc. and can even be manipulated with the mind. In the future of display design, the addition of digital media art will certainly make it shine. The future of display design will be more diverse in form and richer in content. It is believed that in recent years, digital media art in display design will be the main character, and its powerful advantages can make the traditional exhibits new and give people a new sense of enjoyment, and in the context of the development of the times, but also more in line with people's aesthetics and needs, as shown in Figure 8.

The development of new media technology has made traditional things more efficient and accessible. To create $3 \mathrm{D}$ character animation with the character and to create digital sculptures with convenience require the creator to delve deeper into the commonalities between the two and to find the connections between them. This way there is not only basic knowledge at the theoretical level but also indepth research at the technical level. Not only because the software can interoperate with each other but also because any frame in the movement of the animation character is a complete digital sculpture scene, and the creation of digital sculpture will choose the most beautiful, smooth, and expressive frame in the animation frame as a reference for creation. The development of 3D technology allows the work to recreate according to the environmental and stylistic characteristics of modern public space. The artist's concept and technical expression will be adapted as much as possible to the needs of the audience, satisfy-

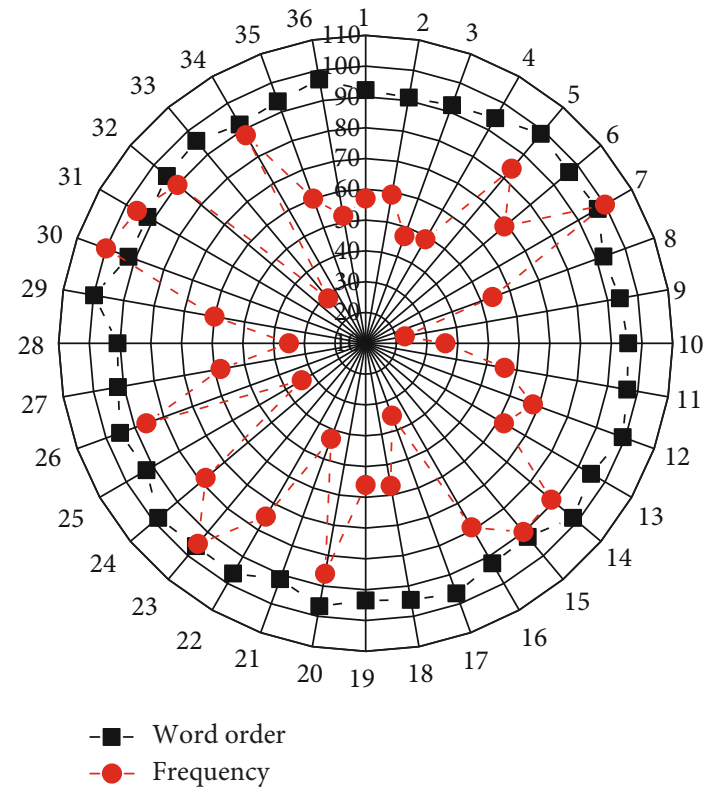

Figure 8: Preposition frequency reduction results.

ing their appreciation, entertainment, and satisfaction with the work. Find connections between the disciplines based on the similarities between 3D character animation and digital sculpture.

\section{Conclusion}

Based on the data support provided by the corpus, a semantic description method is used to generate character animations, and then $3 \mathrm{D}$ animations are displayed in the form of an improved digital media player to realize the design of a corpus-based 3D animation digital media system. The experiments prove that the system in this paper can effectively generate $3 \mathrm{D}$ animation based on text content, while the corpus module can provide higher accuracy for data extraction, and the improved digital media player can realize the display of animation on different platforms. With the increasing application of computer technology in university classrooms, the existing problems are gradually exposed. This article summarizes and discusses the reasons for the application of computer technology in university classrooms and proposes solutions to this problem. Countermeasures provide a reference for the application of computer technology in university teaching classrooms. Data collection, system architecture, data flow, database design, and system testing are all included in this complex management project. For the design company, the design of the whole system of $3 \mathrm{D}$ animation material, to combine the big theoretical approach to its practical situation, this paper has limitations in some viewpoints and issues due to the fineness of the research; thus, the $3 \mathrm{D}$ animation material resource database, the connotation of the resource platform, and the system construction issues cannot fully include. In the problem of design efficiency improvement, it is still necessary to keep exploring and deep thinking, so that the connotation of this paper can be expanded indepth. 


\section{Data Availability}

The data used to support the findings of this study are included within the article.

\section{Conflicts of Interest}

No competing interests exist concerning this study.

\section{References}

[1] J. McDonald, R. Wolfe, S. Baowidan, N. Guo, S. Johnson, and R. Moncrief, "Using N-gram analytics to improve automatic fingerspelling Generation," Linguistics and Literature Studies, vol. 5, no. 3, pp. 187-197, 2017.

[2] X. Liu, J. Lyu, and D. Zheng, "For a better dictionary: revisiting ecolexicography as a new paradigm," Lexikos, vol. 31, no. 1, pp. 283-321, 2021.

[3] J. Fernández-Cruz and A. Moreno-Ortiz, "Building the great recession news corpus (GRNC): a contemporary diachronic corpus of economy news in English," Research in Corpus Linguistics, vol. 8, no. 2, pp. 28-45, 2020.

[4] J. Zakraoui, M. Saleh, and J. Al Ja'am, "Text-to-picture tools, systems, and approaches: a survey," Multimedia Tools and Applications, vol. 78, no. 16, pp. 22833-22859, 2019.

[5] J. M. de Martino, I. R. Silva, C. Z. Bolognini et al., "Signing avatars: making education more inclusive," Universal Access in the Information Society, vol. 16, no. 3, pp. 793-808, 2017.

[6] P. Mathur and H. Mathur, "A study on speech recognition techniques in regional languages," International Journal on Future Revolution in Computer Science \& Communication Engineering, vol. 4, no. 11, pp. 110-114, 2018.

[7] G. Eryiğit, C. Eryiğit, S. Karabüklü et al., "Building the first comprehensive machine-readable Turkish sign language resource: methods, challenges and solutions," Language Resources and Evaluation, vol. 54, no. 1, pp. 97-121, 2020.

[8] X. Wen, M. Wang, C. Richardt, Z. Y. Chen, and S. M. Hu, "Photorealistic audio-driven video portraits," IEEE Transactions on Visualization and Computer Graphics, vol. 26, no. 12, pp. 3457-3466, 2020.

[9] S. Neumann, "On the interaction between register variation and regional varieties in English," Language, Context and Text, vol. 2, no. 1, pp. 121-144, 2020.

[10] J. Françoise and F. Bevilacqua, "Motion-sound mapping through interaction," ACM Transactions on Interactive Intelligent Systems (TiiS), vol. 8, no. 2, pp. 1-30, 2018.

[11] X. Wang, W. Chang, and X. Tan, "Representing and linking Dunhuang cultural heritage information resources using knowledge graph," KO KNOWLEDGE ORGANIZATION, vol. 47, no. 7, pp. 604-615, 2020.

[12] J. Aydınlı and D. Ortaçtepe, "Selected research in applied linguistics and English language teaching in Turkey: 20102016," Language Teaching, vol. 51, no. 2, pp. 210-245, 2018.

[13] M. Chollet, M. Ochs, and C. Pelachaud, "A methodology for the automatic extraction and generation of non-verbal signals sequences conveying interpersonal attitudes," IEEE Transactions on Affective Computing, vol. 10, no. 4, pp. 585-598, 2019.

[14] K. Feher, "Recommendations, best practices and key factors of smart city and smart citizenship. Smart cities and regional development (SCRD)," Journal, vol. 4, no. 1, pp. 37-55, 2020.
[15] Y. Gambier and H. Jin, "A connected history of audiovisual translation," Translation Spaces, vol. 8, no. 2, pp. 193-230, 2019.

[16] Y. Wang and Y. Zhou, "Systemic-functional linguistics in China (2010-2016),” WORD, vol. 64, no. 1, pp. 9-37, 2018.

[17] J. Kaplan and R. Lemov, "Archiving endangerment, endangered archives: journeys through the sound archives of Americanist anthropology and linguistics, 1911-2016," Technology and Culture, vol. 60, no. 2S, pp. S161-S187, 2019.

[18] C. Martins and C. Ferreira, "Project-based learning in audiovisual translation," Journal of Audiovisual Translation, vol. 2, no. 1, pp. 152-182, 2019.

[19] Y. Gu, C. Wang, J. Ma, R. J. Nemiroff, D. L. Kao, and D. Parra, "Visualization and recommendation of large image collections toward effective sensemaking," Information Visualization, vol. 16, no. 1, pp. 21-47, 2017.

[20] B. Michelson, "The Year's work in American humor studies, 2017," Studies in American Humor, vol. 5, no. 1, pp. 157242, 2019.

[21] T. Grubljesic, P. S. Coelho, and J. Jaklic, "The shift to socioorganizational drivers of business intelligence and analytics acceptance," Journal of Organizational and End User Computing, vol. 31, no. 2, pp. 37-64, 2019.

[22] M. Lin, Y. Chen, and R. Chen, "Bibliometric analysis on Pythagorean fuzzy sets during 2013-2020," International Journal of Intelligent Computing and Cybernetics, vol. 14, no. 2, pp. 104-121, 2021. 